\title{
Variação morfológica em populações de Tetragonopterus argenteus \\ Cuvier, 1817 (Characiformes, Characidae) das bacias dos \\ rios Madeira e Paraguai
}

\section{Morphological variation in populations of Tetragonopterus argenteus Cuvier, 1817 (Characiformes, Characidae) from Madeira and Paraguay river basins}

\author{
Oscar Akio Shibatta ${ }^{1}$; Willian Massaharu Ohara $^{2}$
}

Resumo

O sauá Tetragonopterus argenteus é endêmico da América do Sul, apresenta ampla distribuição geográfica e ocorre em várias bacias brasileiras, entre elas as dos rios Madeira e Paraguai. Neste trabalho foi observado que as populações dessas bacias apresentam variações morfológicas que, provavelmente, representam isolamento geográfico. Exemplares foram estudados através de 12 caracteres morfológicos e submetidos às análises de variáveis canônicas e de proporções corporais. Na análise das variáveis canônicas, as populações das bacias dos rios Madeira e Paraguai diferenciaram-se pelo diâmetro do olho, distância pré-ventral, altura do corpo, comprimento do focinho, altura do pedúnculo caudal e comprimento da cabeça. Na análise das proporções corporais, a população da bacia do rio Madeira apresentou comprimento da cabeça, diâmetro do olho, distância pré-dorsal, comprimento da base da nadadeira dorsal, distância préventral, altura do corpo e altura do pedúnculo caudal, menores que os das populações da bacia do rio Paraguai. Esses resultados possibilitam o reconhecimento da amplitude da variação intra-específica, que poderá ser um importante instrumento para o manejo e conservação dessa espécie de peixe.

Palavras-chave: Região Neotropical. Peixes. Tetragonopterinae. Morfometria multivariada. Endêmico.

\begin{abstract}
The saua Tetragonopterus argenteus is endemic to South America. It has a wide geographic distribution and occurs in different Brazilian basins, such as those of the Madeira and Paraguay rivers. In this work, it was noticed that populations from these basins have morphological variations between them, indicating a possible geographic isolation. Specimens were studied using twelve morphological characters that were submitted to the canonical variates and body proportion analysis. In the canonical variate analysis, the populations from the Madeira and Paraguay river basins were differentiated by caudal peduncle depth, eye diameter, pre-ventral distance, body height, snout length and head length. In the body proportion analysis, the population of Madeira river basin presented head length, orbital diameter, pre-dorsal distance, dorsal-fin length, pre-ventral distance, body height and caudal peduncle height, smaller than those of the population from Paraguay river basin. These results allowed the recognition of intra-specific variation amplitude, that could become an important instrument for management and conservation of this species.
\end{abstract}

Key words: Neotropical Region. Fishes. Tetragonopterinae. Multivariate morphometry. Endemic.

\footnotetext{
1 Professor Associado do Departamento de Biologia Animal e Vegetal, Universidade Estadual de Londrina, Universidade Estadual de Londrina. Londrina, PR. E-mail: shibatta@uel.br.

2 Graduando do Curso de Ciências Biológicas, Universidade Estadual de Londrina.
} 


\section{Introdução}

Os characiformes constituem uma das ordens dominantes e de maior diversidade entre os peixes tropicais. A ordem é composta por cerca de 1.300 espécies, distribuídas em 16 famílias e 230 gêneros (OYAKAWA et al., 2006). Muitas espécies são endêmicas da região Neotropical, mas poucas se encontram no continente africano e na América Central. As principais características utilizadas para identificar peixes dessa ordem são escamas cobrindo todo o corpo, com exceção da cabeça; presença de nadadeira adiposa; raios das nadadeiras moles e prémaxilar fixo ao crânio (OYAKAWA et al., 2006).

Characidae é a família mais numerosa desta ordem, com 960 espécies. Apesar dessa alta diversidade, a sistemática ainda continua uma incógnita (BRITSKI, 1992), pois poucas relações filogenéticas estão sustentadas por caracteres nãoambíguos. A subfamília Tetragonopterinae era considerada como um dos grupos de peixes mais importantes do Brasil (BRITSKI, 1972), assertiva reforçada pelo grande número de táxons (50 gêneros e 450 espécies) distribuídos por toda região Neotropical (GÉRY, 1977). Entretanto, devido aos problemas com a sistemática do grupo, atualmente ela abriga apenas o gênero Tetragonopterus (Cuvier, 1817) (REIS; KULLANDER; FERRARIS, 2003). Esse gênero incluía aproximadamente 130 espécies antes da revisão de Eigenmann (1917), que transferiu muitas espécies para os gêneros Astyanax, Moenkhausia e Bryconamericus (GÉRY, 1977).

Endêmico da América do Sul, o gênero Tetragonopterus está presente em muitas bacias brasileiras. Possui cinco espécies nominais e quatro espécies válidas: T. argenteus Cuvier, 1817, T. chaceus Agassiz, 1829, T. hunberi Steindachner, 1909 e T. lemniscatus Benine, 2004, além de uma espécie ainda não descrita proveniente das cachoeiras do rio Xingu, no Estado do Pará (PELIÇÃO, 2001).
Segundo Pelição (2001), o Tetragonopterus argenteus (Figura 1) é caracterizado pelo: corpo alto e comprimido sendo sua maior altura em frente ao primeiro raio da nadadeira dorsal; diâmetro do olho relativamente grande; boca pouco fendida; duas fileiras de dentes no pré-maxilar sendo, a interna com cinco dentes; área pré-ventral achatada; perfil dorsal do corpo acentuadamente côncavo; perfil do pedúnculo caudal côncavo; perfil ventral da cabeça convexo; perfil ventral do início da nadadeira anal até o pedúnculo caudal formando um ângulo de $45^{\circ}$ até a nadadeira pélvica convexo; perfil ventral da nadadeira pélvica até a nadadeira anal reto; primeira fileira dorsal de escamas pouco definida; nadadeira anal longa e linha lateral descendo abruptamente após o opérculo. Apresenta uma ampla distribuição geográfica, pois ocorre nas bacias dos rios Amazonas, Paraná-Paraguai, Orinoco e região oeste do nordeste do Brasil (PELIÇÃO, 2001). Entretanto, foi observado que as populações da bacia do rio Madeira e Paraguai apresentam variações morfológicas que provavelmente representam variações geográficas (OHARA; SHIBATTA; BENINE, 2006). Desta forma, este trabalho teve o objetivo de levantar caracteres morfométricos e merísticos que permitissem reconhecer essas diferenças populacionais.

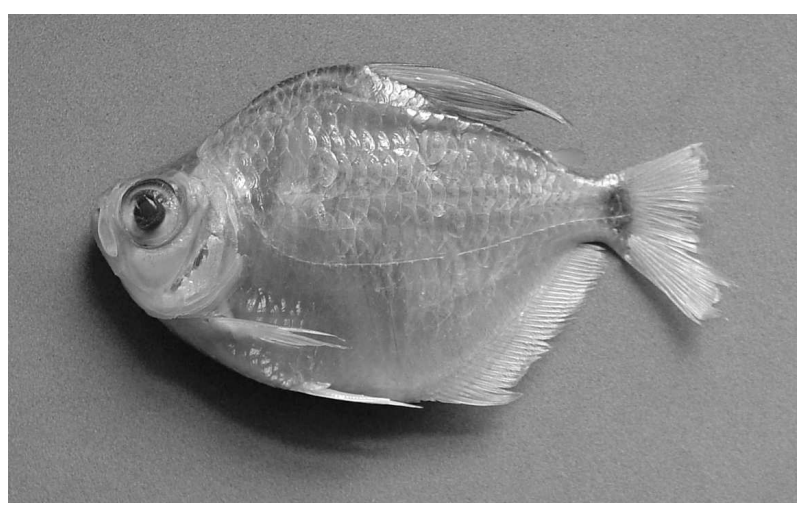

Figura 1. Tetragonopterus argenteus, MZUEL 3810, rio Salobo, Porto Estrela, MT, 01-03.vii.1999, 62,45 mm de comprimento padrão. 


\section{Material e Métodos}

Para as 3 contagens (Tabela 1) e 12 medidas (Tabela 2), foram utilizados, respectivamente, um microscópio estereoscópio e um paquímetro digital com precisão de $0,01 \mathrm{~mm}$, segundo metodologia proposta por Fink e Weitzman (1974). As medidas relacionadas ao corpo foram apresentadas como proporção do comprimento padrão, e as da cabeça, em relação ao comprimento da cabeça. Para análise multivariada dos dados morfométricos das variáveis canônicas livres do tamanho, foi utilizado o programa PAST (HAMMER; HARPER; RYAN, 2003), opção MANOVA/CVA sobre os dados normalizados e logaritmizados.

O material examinado está depositado no Museu de Zoologia da Universidade Estadual de Londrina e são provenientes de diversas localidades: bacia do rio Madeira, afluentes do rio Ji-Paraná [7 exemplares, 2 exs. do rio Tamarupá (MZUEL 4512, município de
Cacoal, RO, col. L. Perin, 06.vi.2005, 1 ex..; MZUEL 4459, mun. Cacoal, RO, col. L. Perin, 03.vi.2005, 1 ex.) e 5 exs. do rio Pirarara (MZUELl 4510, mun. Cacoal, RO, col. L. Perin, 27.ii.2005, 1 ex.; MZUEL 4508, mun. Cacoal, RO, col. L. Perin, 28.v.2005, 1 ex.;MZUEL 4511, mun. Cacoal, RO, col. L. Perin, 26.ii.2005, 2 exs.; MZUEL 4509, mun. Cacoal, RO, col. L. Perin, 29.v.2005, 1ex.)] e da bacia do rio Paraguai [24 exs., 3 exs. do rio Pixaim, bacia do rio Cuiabá (MZUEL 4454, mun. Poconé, MT, col. O. A. Shibatta, 09.vii. 2000), 4 exs. do rio Salobo, afluente do rio Paraguai (MZUEL 3810, mun. Porto Estrela, MT, col. O. A. Shibatta, 01-03. vii.1999) e 7 exs. do rio Miranda/ Bonito (MZUEL 4445, mun. Bonito, MS, col. M. C. Filho, 19-26.x.1999) e 10 exs. do rio Miranda/ Passo do Lontra ( MZUEL 1390, mun. Corumbá, MS, col. M. C. Filho, v.1991, 7 exs.; MZUEL 4416, mun. Corumbá, MS, COL. M. C. Filho 16-26.ix.1998, 3 ex.)] (Figura 2).
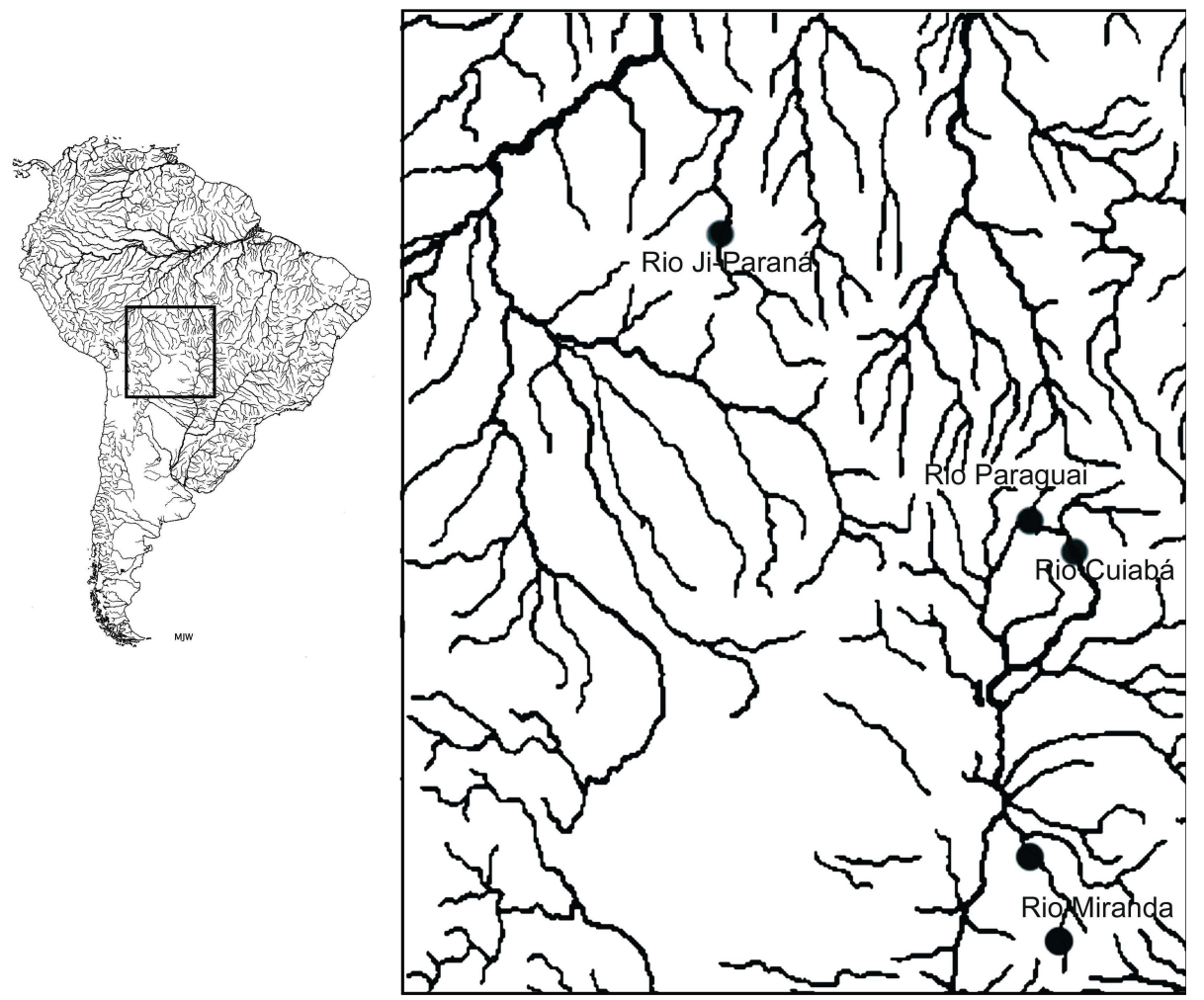

Figura 2. Localidades amostradas de Tetragonopterus argenteus nos rios Pirarara e Tamarupá (bacia do Rio JiParaná), Rio Salobo (bacia do alto Rio Paraguai), Rio Pixaim (bacia do Rio Cuiabá) e o Rio Miranda [Bonito (ao sul) e Passo do Lontra (ao norte). 


\section{Resultados}

Neste trabalho, o número de raios da nadadeira anal variou entre 36 a 41, da nadadeira peitoral entre
13 a 15 e da linha lateral entre 32 a 34 escamas perfuradas (Tabela 1).

Tabela 1. Caracteres merísticos das populações de Tetragonopterus argenteus das bacias do Madeira e Paraguai. Mín. = mínimo, máx. $=$ máximo, $\mathrm{n}=$ número de exemplares examinados.

\begin{tabular}{l|l|l|l|l|l|l|l|l|l|l}
\hline & \multicolumn{3}{|c|}{$\begin{array}{c}\text { Bacia do rio } \\
\text { Madeira }(\mathrm{n}=7)\end{array}$} & \multicolumn{2}{c|}{$\begin{array}{c}\text { Rio Miranda/Passo do } \\
\text { Lontra }(\mathrm{n}=10)\end{array}$} & $\begin{array}{c}\text { Rio Miranda/Bonito } \\
(\mathrm{n}=7)\end{array}$ & \multicolumn{3}{c|}{$\begin{array}{c}\text { Rio Pixaim } \\
(\mathrm{n}=3)\end{array}$} & \multicolumn{2}{c}{$\begin{array}{c}\text { Rio Salobo } \\
(\mathrm{n}=4)\end{array}$} \\
\hline & Mín-máx & Moda & Mín-máx & Moda & Mín-máx & Moda & Mín-máx & Moda & Mín-máx & Moda \\
\hline Escamas da linha lateral & $32-34$ & 33 & $33-34$ & 33 e 34 & $33-34$ & 33 & $32-33$ & 33 & $33-34$ & 33 \\
\hline Raios da nadadeira anal & $36-39$ & 36 & $37-41$ & 38 & $36-38$ & 36 & $36-40$ & 36,39 e 40 & $38-40$ & 38 \\
\hline $\begin{array}{l}\text { Raios da nadadeira } \\
\text { peitoral }\end{array}$ & $13-15$ & 14 & $13-14$ & 14 & $13-15$ & 15 & $14-14$ & 14 & $14-15$ & 15 e14 \\
\hline
\end{tabular}

Na análise de proporções corporais (Tabela 2), é possível notar que as populações da bacia do rio Paraguai não podem ser distinguidas entre si, mas elas, em conjunto, diferem da população da bacia do rio Madeira. As populações do rio Paraguai apresentaram o comprimento da cabeça, diâmetro do olho, distância pré-dorsal, comprimento da base da nadadeira dorsal, distância pré-ventral, altura do corpo e altura do pedúnculo caudal ligeiramente maiores, quando comparados com a população do rio Madeira. Já a distância interorbital, comprimento do focinho, largura da boca e distância entre nadadeira anal e ventral são semelhantes entre essas populações.

Tabela 2. Proporções corporais das populações de Tetragonopterus argenteus das bacias dos rios Madeira $(\mathrm{n}=7)$, Miranda/ Passo do Lontra $(n=10)$, Miranda/Bonito $(n=7)$ Pixaim $(n=3)$ e Salobo $(n=4)$. Medidas relacionadas ao corpo como porcentagens do comprimento padrão e as relacionada à cabeça, em relação ao comprimento da cabeça.

\begin{tabular}{|c|c|c|c|c|c|c|c|c|c|c|}
\hline \multirow{2}{*}{ Mín = mínimo; máx= máx } & \multicolumn{4}{|c|}{ 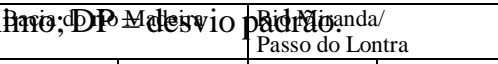 } & \multicolumn{2}{|c|}{ Rio Miranda/Bonito } & \multicolumn{2}{|l|}{ Rio Pixaim } & \multicolumn{2}{|l|}{ Rio Salobo } \\
\hline & Mín.-máx. & Média \pm DP & Mín.-máx. & Média \pm DP & Mín.-máx. & Média \pm DP & Mín.-máx. & Média $\pm \mathrm{DP}$ & Mín.-máx. & Média $\pm \mathrm{DP}$ \\
\hline \multicolumn{11}{|l|}{$\begin{array}{l}\text { Porcentagens do comprimento da } \\
\text { cabeça }\end{array}$} \\
\hline Comprimento da cabeça & $23,1-29,5$ & $27,0 \pm 2,0$ & $28,1-30,9$ & $29,4 \pm 0,9$ & $27,4-28,9$ & $27,9 \pm 0,7$ & $30,9-34,4$ & $32,8 \pm 1,8$ & $29,5-31,5$ & $30,6 \pm 0,9$ \\
\hline Diâmetro do olho & $39,9-44,4$ & $43,1 \pm 1,6$ & $42,2-48,0$ & $45,7 \pm 1,9$ & $41,1-46,7$ & $44,1 \pm 2,1$ & $45,3-48,1$ & $46,7 \pm 1,4$ & $43,1-47,8$ & $45,3 \pm 1,9$ \\
\hline Distância interorbital & $31,1-43,3$ & $39,4 \pm 3,9$ & $36,1-42,0$ & $39,0 \pm 2,0$ & $37,1-43,9$ & $41,8 \pm 2,2$ & $31,3-36,4$ & $33,8 \pm 2,5$ & $36,4-39,0$ & $37,7 \pm 1,0$ \\
\hline Comprimento do focinho & $22,2-33,5$ & $26,7 \pm 3,8$ & $23,3-28,6$ & $26,0 \pm 1,7$ & $26,2-30,7$ & $28,6 \pm 1,5$ & $22,1-24,0$ & $23,1 \pm 1,0$ & $24,9-27,1$ & $25,8 \pm 0,9$ \\
\hline Largura da boca & $31,1-35,7$ & $33,7 \pm 1,5$ & $28,1-34,9$ & $32,4 \pm 2,0$ & $31,4-35,0$ & $33,1 \pm 1,3$ & $30,0-31,8$ & $31,2 \pm 1,0$ & $30,2-32,0$ & $30,9 \pm 0,8$ \\
\hline \multicolumn{11}{|l|}{$\begin{array}{l}\text { Porcentagens do comprimento } \\
\text { padrão }\end{array}$} \\
\hline Distância pré-dorsal & $48,3-55,0$ & $53,3 \pm 2,3$ & $53,0-56,3$ & $54,7 \pm 1,0$ & $54,2-58,9$ & $56,2 \pm 1,5$ & $54,0-57,5$ & $56,1 \pm 1,8$ & $55,2-57,1$ & $56,2 \pm 0,9$ \\
\hline $\begin{array}{l}\text { Comprimento da base da nadadeira } \\
\text { dorsal }\end{array}$ & $14,4-17,7$ & $16,4 \pm 1,1$ & $16,9-18,6$ & $17,8 \pm 0,6$ & $17,0-18,2$ & $17,7 \pm 0,4$ & $17,4-18,7$ & $18,1 \pm 0,6$ & $18,1-19,9$ & $18,8 \pm 0,8$ \\
\hline Distância pré-ventral & $46,8-52,2$ & $50,9 \pm 1,8$ & $48,4-53,5$ & $51,0 \pm 2,0$ & $48,9-51,9$ & $50,6 \pm 1,0$ & $52,8-56,0$ & $54,1 \pm 1,7$ & $50,2-52,3$ & $51,1 \pm 0,9$ \\
\hline $\begin{array}{l}\text { Distância entre as nadadeiras } \\
\text { pélvica e anal }\end{array}$ & $20,5-23,9$ & $21,9 \pm 1,1$ & $20,0-23,4$ & $22,1 \pm 1,1$ & $23,0-27,6$ & $24,9 \pm 1,6$ & $22,6-24,5$ & $23,4 \pm 1,0$ & $22,6-26,0$ & $24,4 \pm 1,4$ \\
\hline Altura do corpo & $53,9-60,9$ & $58,3 \pm 2,5$ & $58,2-65,6$ & $60,6 \pm 2,5$ & $61,2-66,0$ & $63,0 \pm 1,6$ & $63,8-64,5$ & $64,1 \pm 0,4$ & $62,7-64,9$ & $64,3 \pm 1,1$ \\
\hline Altura do pedúnculo caudal & $8,9-11,1$ & $10,4 \pm 0,8$ & $10,8-11,9$ & $11,5 \pm 0,4$ & $11,4-12,3$ & $11,9 \pm 0,3$ & $11,2-11,6$ & $11,4 \pm 0,2$ & $11,7-12,5$ & $12,1 \pm 0,3$ \\
\hline
\end{tabular}

$\mathrm{Na}$ análise das variáveis canônicas, o eixo canônico 1 reteve $62,6 \%$ da variância dos dados originais e o eixo 2 reteve $24,9 \%$. Pelo Gráfico 1 , podemos observar que a população da bacia do rio Madeira ficou discriminada das populações da bacia do rio Paraguai, no eixo 1. As variáveis que contribuíram para a separação desses dois grupos maiores foram o diâmetro do olho, a altura do corpo, o comprimento do focinho, a altura do pedúnculo caudal, comprimento da cabeça, comprimento da 
base da nadadeira dorsal e distância entre as nadadeiras pélvica e anal (Tabela 3) que foram maiores nos peixes da bacia do rio Paraguai (Tabela 2). Na população do rio Madeira, salientaram-se os valores da distância pré-ventral, comprimento padrão e distância pré-dorsal.

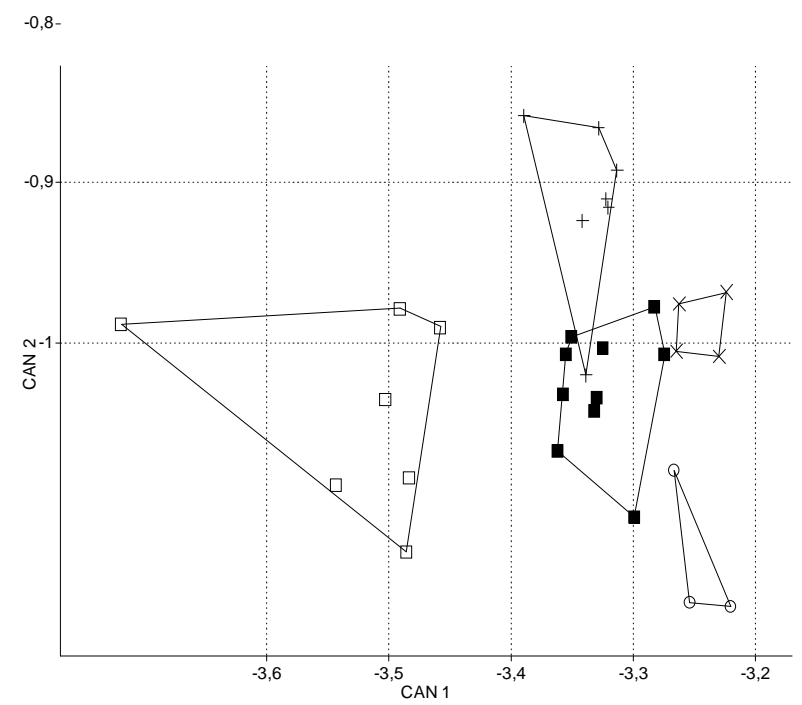

Gráfico 1. Variáveis Canônicas dos exemplares de Tetragonopterus argenteus da bacia do rio Madeira (quadrados claros), Miranda/Bonito (+), Miranda/Passo do Lontra (quadrados escuros), Pixaim (círculos claros) e Salobo (X).

\section{Discussão}

Pode-se observar que os valores dos números de raios das nadadeiras anal e peitoral observados por Pelição (2001) sobrepuseram-se aos das populações analisadas neste trabalho. Entretanto, o mesmo não ocorreu para o número de escamas perfuradas da linha lateral, que apresentou menores valores em Pelição (2001) (27 a 32 raios vs. 32 a 34).

As diferenças morfométricas entre as populações de Tetragonopterus argenteus da bacia do rio Paraguai e do rio Madeira podem ser resultado das divergências morfológicas acumuladas ao longo de sua história evolutiva, em função do isolamento geográfico e reprodutivo conferido pelo Planalto Matogrossense. Esse planalto, subdivisão do planalto brasileiro, serve de divisor de águas entre os rios que correm para bacia do Paraguai e os rios da bacia do rio Amazonas (CUNHA, 1998).

Levantar diferenças entre populações pode ser importante para a conservação das espécies, à medida que permite reconhecer a amplitude de diversidade dentro da espécie (SHIBATTA; HOFFMANN, 2005). As diferenças morfométricas observadas podem ser conseqüências das características genéticas e de diferentes pressões ecológicas. As pressões ambientais, entretanto,

Tabela 3. Pesos das variáveis nos eixos canônicos 1 e 2 obtidos pela análise das variáveis canônicas livres do tamanho das amostras combinadas de Tetragonopterus argenteus das bacias dos rios Madeira e Paraguai.

\begin{tabular}{l|c|c}
\hline & Eixo1 & Eixo2 \\
\hline Comprimento padrão & $-0,23098$ & 0,30389 \\
\hline Comprimento da cabeça & 0,30503 & $-0,3801$ \\
\hline Diâmetro do olho & 0,46825 & $-0,37432$ \\
\hline Distância interorbital & 0,18015 & 0,198 \\
\hline Comprimento do focinho & 0,31719 & 0,17323 \\
\hline Largura da boca & $-0,00923$ & $-0,11775$ \\
\hline Distância pré-dorsal & $-0,17813$ & 0,26686 \\
\hline Comprimento da base da nadadeira dorsal & 0,24311 & 0,11084 \\
\hline Distância pré-ventral & $-0,39507$ & $-0,20283$ \\
\hline Distância entre as nadadeiras pélvica e anal & 0,22915 & 0,29187 \\
\hline Altura do corpo & 0,327 & $-0,09545$ \\
\hline Altura do pedúnculo caudal & 0,30796 & 0,5666 \\
\hline
\end{tabular}


provavelmente não estão fortemente relacionadas à velocidade da água, pois a altura do corpo não foi o fator que determinou a diferenciação das populações. Segundo Breda, Oliveira e Goulart (2005), diferenças intra-específicas na altura do corpo podem ser observadas em peixes que vivem em ambiente lótico, que têm menor altura do que os que vivem em ambientes lênticos. Mesmo assim, a população do rio Madeira se salientou pelo maior comprimento da região anterior do corpo, antes das nadadeiras dorsal e pélvica, o que pode significar ligeira diferença na sua hidrodinâmica. Entretanto, as três características mais importantes que diferenciaram as populações do rio Paraguai da do rio Madeira, com exceção da altura do pedúnculo caudal, estão relacionadas à cabeça (diâmetro do olho, comprimento do focinho e da cabeça), ou seja, com a percepção visual do ambiente.

As diferenças ocorridas entre os pesos de algumas variáveis na discriminação das populações do rio Paraguai e do rio Madeira, provavelmente, são problemas decorrentes dos erros acumulados entre as duas variáveis, quando se calculam as proporções corporais, o que não ocorre com a aplicação da análise estatística multivariada. Portanto, a aplicação de análises estatísticas multivariadas é mais adequada à natureza multidimensional dos dados morfométricos, conforme já obserado por Reis, 1988.

\section{Agradecimentos}

Os autores agradecem a Paulo Bernardes e Leane Perin pela doação dos exemplares da bacia do rio Madeira. Ao Ricardo C. Benine pelas valiosas discussões e informações. A Leonardo Dagui de Oliveira pela ajuda no abstract. À Universidade Estadual de Londrina pelo apoio de infra-estrutura laboratorial, assim como pelo apoio financeiro das coletas dos exemplares da bacia do rio Paraguai, efetuadas durante a realização da disciplina Biologia de Campo.

\section{Referências}

BRITSKI, H. A. Peixes da água doce do Estado de São Paulo: sistemática. In: COMISSÃO INTERESTADUAL ESTADUAL DA BACIA PARANÁ-URUGUAI. Poluição e piscicultura. São Paulo: Faculdade de Saúde Pública da USP, Instituto de Pesca, 1972. p.70-108.
BRITSKI, H. A. Conhecimento atual das relações filogenéticas de peixes neotropicais. In: ENCONTRO BRASILEIRO DE ICTIOLOGIA, 4., 1992, Maringá. Anais... Maringá: Universidade Estadual de Maringá, 1992. p.42-57.

BREDA, L.; OLIVEIRA, E. F.; GOULART, E. Ecomorfologia de locomoção de peixes com enfoque para espécies neotropicais. Acta Scientiarum Biological Sciences, Maringá, v.27, n.4, p.371-381, 2005.

CUNHA, S. B. Bacias hidrográficas. In: CUNHA, S. B.; GUERRA, A. J. T. (Org.) Geomorfologia do Brasil. São Carlos: EDUSP, 1998. p.229-271.

EIGENMANN, C. H. The American Characidae. Memoirs of the Museum of Comparative Zoology, Havard, v.53, n.1, p.1-102, 1917.

GÉRY, J. Characoids of the world. Neptune: TFH Publications, 1977.

FINK, W. F; WEITZMAN, S. H. The So-called Cheirodontin Fishes of Central America with description of two new species (Pisces: Characidae). Smithsonian Contributions to Zoology, Washington, v.172, p.1-46, 1974.

HAMMER, O.; HARPER, D. A. T.; RYAN, P. D. PASTPalaeontological Statistic. Disponível em: <http:// folk.uio.no/ohammer/past>. Acesso em: 31 jul. 2003.

OHARA, W. M.; SHIBATTA, O. A.; BENINE, R. C. Variação Geográfica em populações de Tetragonopterus argenteus Cuvier, 1816 (Characiformes, Tetragonopteridae). CONGRESSO BRASILEIRO DE ZOOLOGIA, 26., 2006, Londrina. Resumos... Londrina: Sociedade Brasileira de Zoologia, 2006.

OYAKAWA, O. T.; AKAMA, A.; MAUTARI, K. C.; NOLASCO. J. C. Peixes de riachos da Mata Atlântica nas unidades de Conservação do Vale do rio Ribeira de Iguape no Estado de São Paulo. São Paulo: Neotropica, 2006.

PELIÇÃO, G. Z. Taxonomia e relações filogenéticas de Tetragonopterus Cuvier, 1817 (Characiformes, Characidae). 2001. Dissertação (Mestrado) - Faculdade de Filosofia Ciências e Letras de Ribeirão Preto/USP, Ribeirão Preto.

REIS, S. F. Morfometria e estatística multivariada em biologia evolutiva. Revista Brasileira de Zoologia, São Paulo, v.5, n.4, p.571-580, 1988.

REIS, R. E.; KULLANDER, S. O.; FERRARIS, C. J. Check list of the freshwater fishes of South and Central America. Porto Alegre: EDIPUCRS, 2003.

SHIBATTA, O. A.; HOFFMANN, A. C. Variação geográfica em Corydoras paleatus (Jenyns) (Siluriformes, Callichthyidae) do sul do Brasil. Revista Brasileira de Zoologia, São Paulo, v.22, n.2, p.366-371, 2005. 